\title{
Birdsongs alleviate anxiety and paranoia in healthy participants.
}

\author{
Stobbe $^{1}$, E., Sundermann ${ }^{1}$, J., \& Ascone ${ }^{2}$, L. \& Kühn ${ }^{1,2}$, S. \\ S. Kühn \& L. Ascone share last authorship
}

${ }^{1}$ Max Planck Institute for Human Development, Lise Meitner Group for Environmental Neuroscience, Lentzeallee 94, 14195 Berlin, Germany

${ }^{2}$ University Medical Center Hamburg-Eppendorf, Department of Psychiatry and

Psychotherapy, Neuronal Plasticity Working Group, Martinistr. 52, 20246, Hamburg, Germany. Contact information of corresponding author: stobbe@mpib-berlin.mpg.de 


\begin{abstract}
The present study investigated the effect of urban (traffic noise) vs. natural (birdsongs) soundscapes on mood, state paranoia, and cognitive performance, hypothesizing that birdsongs lead to significant improvements in these outcomes. An additional goal was to examine the differential impact of lower vs. higher diversity of the soundscapes by manipulating the number of different typical traffic sounds or songs of different bird species within the respective soundscapes. In a randomized online experiment, 295 participants were exposed to one out of four conditions for six minutes: traffic noise low, traffic noise high, birdsong low, and birdsong high diversity soundscapes. Before and after the exposure, participants performed a digit-span and dual n-back task, and filled out depression, anxiety, and paranoia questionnaires. The traffic noise soundscapes were associated with a significant increase in depression (small effect size in low, medium effect size in high diversity condition). Concerning the birdsong conditions, depression exclusively decreased after exposure to the high diversity soundscape (small effect size). Anxiety and paranoia significantly improved in both birdsong conditions (medium effect size). For cognition, no effects were observed. In sum, the present study shows that listening to birdsongs improves anxiety, while traffic noise is generally related to higher depressiveness, regardless of diversity. Moreover, for the first time, a beneficial, medium-sized effect of a natural birdsong soundscape was demonstrated for paranoia, which bears interesting implications for further research in paranoia-prone individuals or even psychiatric samples.
\end{abstract} Keywords: paranoia, anxiety, depression, birdsongs, traffic noise, psychological restoration 


\section{Introduction}

The impact of the environmental on psychological well-being and cognition in humans have for a long time been neglected in traditional psychology. At present, human living environments are changing drastically. According to the UN 2007 was a turning point for humankind as for the first time the majority of the global population lived in urban areas (Ritchie \& Roser, 2018). Until 2050, it is estimated that $68 \%$ of the world population will be living in cities (68\% of the World Population Projected to Live in Urban Areas by 2050, Says UN|UN DESA | United Nations Department of Economic and Social Affairs, 2018). In Europe the urbanization rate is already as high as $75 \%$. Urbanization coincides with increasing rates of mental illness. An earlier review from 2005 came to the conclusion that about $30 \%$ of the incidence in schizophrenia may be attributed to urban factors in interaction with genetic liability and social adversity (Krabbendam, 2005). A recent review on depression and urbanicity reported mixed results, however with the majority of studies suggesting an elevated risk of depression in more (vs. less) urbanized areas (Sampson et al., 2020). Concerning mood and anxiety disorders, a review on studies conducted in Europe constitutes that most studies reported elevated risks of mood/ anxious symptoms when comparing some (not all) of the urban with rural areas (Penkalla \& Kohler, 2014). In sum, there is accumulating evidence that living in urban areas is related to worse mental health outcomes.

One notion is that man-made urban soundscapes (the so-called anthrophone) constitute constant stressors that impair cognitive function and well-being. On the other hand, natural sounds such as birdsongs, wind and water could be an important source of attentionrestorative and stress-ameliorating effects. Stress reduction theory (SRT) posits that humans have an innate positive drive towards interacting with and being in nature, possibly due to its positive 
associations with shelter, food, and rest or recovery from stress (Ulrich, 1983). Birdsong might be implicitly associated with a safe and vital natural environment. On the other hand, urban noise contains salient stimuli that likely trigger an alert, attentive state (e.g., in order to navigate safely on a busy street). Supporting these claims, studies have reported positive effects of birdsongs on human well-being whereby a higher diversity of birds was a decisive factor (Hedblom et al., 2017). A systematic review on traffic noise exposure, found correlative evidence for an associations between traffic noise and depression as well as cognitive decline (Seidler et al., 2019). Similarly, Attention Restoration Theory (ART) states that stimuli from natural sources restore cognitive function by reducing attention demands of the endogenous attention system (Berman et al., 2008). In an experimental study, ART was directly tested by contrasting the effects of urban vs. natural soundscapes. The nature relative to the city sound condition enhanced participants' performance in a dual n-back and digit span task, but did not improve mood (Van Hedger et al., 2019).

The present study broadly builds on the study by van Hedger et al. (Van Hedger et al., 2019) but adds the factor of diversity to the soundscapes. In addition to mood and cognition, the present study focusses on paranoid symptoms, as this is a very prominent symptom in psychosis which can be measured in a change-sensitive manner (Schlier et al., 2016), and which has been shown to be enhanced by building-site noise (Lincoln et al., 2010).

\section{Methods}

The study was pre-registered at aspredicted.org (study name: "Sounds_Online", trial identifier: \#67702, https://aspredicted.org/d5j7j.pdf) on 06/04/2021. A power calculation was conducted for an interaction effect (repeated measures ANOVA), in G*Power 3.1.9.7 with $f=$ $0.10, \alpha=0.05$, power $=.90,4$ groups, correlation between repeated measures $r=0.60$, resulting in a minimum required sample size of $N=288$. 
PSYCHOLOGICAL EFFECTS OF BIRD VS. URBAN SOUNDSCAPES

\subsection{Recruitment and In- and Exclusion Criteria}

The study was programmed using Inquisit 5 (Inquisit 5 [Computer Software], 2016) [https://www.millisecond.com] and accordingly run on the Millisecond server. Participants were recruited from the crowdsourcing platform Prolific and received $10 €$ reimbursement for their full participation. Adult individuals were pre-screened on Prolific (i.e., visibility of the study only for candidates with a suited profile) concerning fluent German language skills (as this was the study language), having no diagnosed lifetime mental illness, and having no hearing difficulties. Prescreened individuals could then access the study, where in- and exclusion criteria were checked further. This included no regular substance or drug intake, no suicidal thoughts, or tendencies, and availability of headphones for the purpose of the study.

\subsection{Sample}

Initially, $N=401$ individuals started the survey. Of those, $n=76$ quit during the sociodemographic assessment, $n=24$ lacked pre-test data, and $n=6$ lacked post-test data. These $n=106$ cases were excluded from the analyses, resulting in a final sample of $N=295$. Of these, some participants had incomplete post-test data ( $n=10$ missing digit span, 5 missing n-back, and $n=8$ missing the qualitative assessments [sound rating]). The participants were in their middle to late twenties on average and there were in tendency more males than females (see Table 1 for details). Positive symptom levels did not differ significantly between the groups. 
Table 1. Descriptive sample data and between-group differences for socio-demographic variables

\begin{tabular}{llllll}
\hline $\begin{array}{l}\text { Variable/ } \\
\text { descriptives }\end{array}$ & $\begin{array}{l}\text { Traffic } \\
\text { noise low } \\
\mathbf{n = 8 3}\end{array}$ & $\begin{array}{l}\text { Traffic } \\
\text { noise high } \\
\mathbf{n = 6 9}\end{array}$ & $\begin{array}{l}\text { Birdsong } \\
\mathbf{l o w} \\
\mathbf{n = 6 3}\end{array}$ & $\begin{array}{l}\text { Birdsong } \\
\text { high } \\
\mathbf{n = 8 0}\end{array}$ & Inferential statistics \\
\hline Age: $M(S D)$ & $27.0(7.48)$ & $25.5(7.10)$ & $26.5(6.30)$ & $28.7(7.72)$ & $F(3,294)=2.53, p=.057$ \\
Sex: \% male & $55 \%$ & $64 \%$ & $71 \%$ & $54 \%$ & $X^{2}(1,294)=6.04, p=.110$ \\
Pos. symptoms & $0.67(0.44)$ & $0.63(0.43)$ & $0.78(0.48)$ & $0.58(0.41)$ & $F(3,294)=2.52, p=.058$ \\
\hline
\end{tabular}

\subsection{Study Procedure}

After providing informed consent, sociodemographic information was assessed, including education, income, living arrangement, and further variables. Psychosis liability was assessed. Hereafter, pre-test assessments were conducted, including an assessment of mood (depression, anxiety), paranoia, the digit-span, and n-back tasks. Participants were randomized to one of four sound conditions: 1) low diversity traffic noise soundscape, 2) high diversity traffic noise soundscape, 3) low diversity birdsong soundscape, or 4) high diversity birdsong soundscape (for details on the stimuli, see Stimuli section). The soundscapes each lasted for exactly 6 minutes. Participants were instructed to set their audio system volume to $80 \%$ (which was piloted with members of our research unit beforehand and deemed to be an optimal average volume) and to listen to the sounds until the end, when participants were required to continue by clicking with their mouse. To assess participants' impatience, the number of clicks during the sound presentation were recorded. In addition, participants were told that a code, consisting of two spoken digits (in German), would be audible towards the end of the sound presentation, which they were required to type in correctly afterwards. This was implemented to assure listening-compliance and attention. After the sound presentation, the pre-test measures were repeated. Finally, several items to assess 
perceived sound quality, including beauty, pleasantness, and monotony (vs. diversity) were presented.

\subsection{Measures}

\subsubsection{Psychosis liability}

Psychosis-liability or sub-clinical psychosis levels was assessed using the Community Assessment of Psychic Experiences (CAPE) (Mossaheb et al., 2012), in its German version, to assesses lifetime positive, negative and depressive symptoms (http://www.cape42.homestead.com/index.html). The CAPE, including the German version, has been validated extensively (Schlier et al., 2015). Items refer to the lifetime prevalence of specific symptoms, rated on an ordinal response scale for frequency (categories: $1=$ 'never', $2=$ 'sometimes', 3 = 'often', 4 = 'nearly always'). The total scale consists of 42 items, whereby the positive symptom scale includes 20 (e.g., 'Do you ever feel as if things in magazines or on TV were written especially for you?'), the negative symptom scale 14 (e.g., 'Do you ever feel that your mind is empty?'), and the depressive symptom scale 8 items (e.g., 'Do you ever feel like a failure?'). To test for comparative baseline levels across all groups in psychosis liability, the positive symptom subscale was used (Mossaheb et al., 2012). It had excellent internal consistency with Cronbach's $\alpha=90$.

\subsubsection{Mood and paranoid symptoms}

Mood was assessed with the State Trait Anxiety Depression Inventory (STADI) (Laux et al., 2016). The scale contains 40 items, whereby the same 20 items are once presented in trait and once in state format. Only the latter was used in the present study. The scale differentiates between depression (low euthymia [inverted items], dysthymia) and anxiety (hyperarousal and worry), whereby each of the subscales is assessed by 5 items. The response format is a 4-point Likert $(1=$ 
'not at all', 4 = 'strongly applies'). Internal consistency (Cronbach's $\alpha$ ) at pre-test was good both for the state anxiety (.85) and depression (.86) scales.

Paranoia was assessed with a brief, change sensitive state version of the paranoia checklist (Schlier et al., 2016). The scale comprises 3 statements (e.g., 'I need to be on my guard against others'), rated on an 11-point Likert-scale (each from 1-11) for the degree of agreement to the statement, associated distress and conviction. The latter two categories were only presented if the rating of agreement to the statement was $>1$ (which accordingly often results in a large amount of missing data). In the present study, only agreement was evaluated. Internal consistency at pre-test was acceptable with Cronbach's $\alpha=.78$.

\subsubsection{Cognition}

To assess digit span cognitive performance, both the forward and backward version were used, as available in Inquisit 5 (Inquisit 5 [Computer Software], 2016) [retrieved from https://www.millisecond.com] which is based on the original task reported by Woods and colleagues (Woods et al., 2011). Two parameters are recommended for evaluation: the two-error maximum length (TE_ML) and the maximum length recalled (ML). Starting with a successive visual presentation of 3 numbers, the participants need to correctly recall an increasing sequence of numbers and reproduce it by clicking the correct numbers in correct order. After two wrongly recalled sequences of the same length, the task is aborted. The participants were explicitly reminded not to use any memory assisting methods such as paper and pencil.

The dual n-back task, also available in the Inquisit 5 (Inquisit 5 [Computer Software], 2016) [retrieved from https://www.millisecond.com] was assessed. The task is based on the original work by Jaeggi and colleagues (Jaeggi et al., 2010). It consists of 4 experimental blocks demanding 2-back and 3-back level performance. While performing the task, subjects pay 
attention to their computer screen while also listening to a computer audio. On each trial a blue square appears in one out of eight grid-like locations around a central fixation cross, while at the same time a (German) letter is presented via the headphones. In the 2-back block condition, the subjects are instructed to press the "A" button on their keyboard when the current square position matches the square position from two trials before. Subjects are also instructed to press the "L" button on their keyboard if the spoken letter matched the letter two trials before. The same instruction, but having to match stimuli 3 trials back, is provided for the 3-back condition. In the present study, participants trained each condition once, and then went on with the experimental blocks. The performance parameter was the so-called $d$ prime value calculated as the proportion of ((visual_TotalHits - visual_TotalFA) +(auditory_TotalHits - auditory_TotalFA)/2)/number of total experimental blocks. Visual hits are defined as correct responses with respect to the location of the square and auditory hits are defined as the correct responses with respect to the spoken letter. False alarms (FA) are defined as responses in the absence of a target.

\subsection{Stimuli}

The soundscapes for all 4 categories have been generated in the same way. Single sound snippets were gathered and then adapted and merged within the audio software Steinberg Cubase10. An exemplary visualization of the resulting soundscape can be seen in the Supplementary Material (Figure SM1). For the nature category a database of birdsong recordings (https://www.xeno-canto.org/explore/region) for the central European region was used. For the low diversity birdsong condition, eight recordings from the same two species were used (common chiffchaff \& wood warbler). For the high diversity birdsong condition, the same approach was chosen, but recordings from eight different bird species were used to create the soundscape (garden warbler, honey buzzard, woodlark, Eurasian sparrow hawk, coal tit, greenshank, common crane, 
and black woodpecker). In both birdsong conditions, additionally subtle water and wind sounds were played in the background, to create a constant background of the soundscape. For the traffic noise conditions, sound snippets from eight different car recoding's (https://freesound.org/search/?q=city) were used for the low diversity traffic noise condition while audio-snippets from eight diverse sources of noise pollution associated with the city were used for the high diversity traffic noise condition (ambulance siren, construction, trucks, train, motorcycle, airplane, bus and fire-fighter siren). In both traffic noise soundscapes, a constant subtle traffic flow was audible in the background.

To ensure that all soundscapes were perceived with a similar loudness level, all soundscapes were engineered to have a similar loudness value. The loudness values from all four conditions range between 19.4 and 27.8 LUFS. All soundscapes had a duration of 6 minutes. Prior to the experiment the soundscapes have been presented to a small set of pilot participants rating the similarity of the audio level ensuring a comfortable audio level across all conditions. As a result, at the beginning of the experiment, participants were instructed to set their headphone loudness level to $80 \%$. Soundscapes can be accessed openly via this link https://osf.io/4y $3 \mathrm{vh} /$.

\subsection{Statistical Analyses}

Analyses were run in SPSS 27 (IBM Corp., 2020). To test the effects of high vs. low diverse traffic noise vs. birdsong soundscapes on mood, paranoia, and cognition, several repeated measures analyses of variance (ANOVA) were run to test for group*time interaction effects. The analyses were once run with all participants, and then only with those who entered at least one of the digits (control of compliance of listening to audio, see Study Procedure) correctly, to check for the robustness of findings. Significant interactions identified for any of the outcomes were followed up by post-hoc-tests on two levels. First, within sample t-test for the different 
conditions (i.e., traffic noise low, traffic noise high, birdsong low, birdsong high diversity) were computed. Second, difference scores between diversity categories within conditions were compared (e.g., traffic noise low pre-post difference vs. traffic noise high pre-post difference). To explore mean differences between the qualitative ratings of soundscapes (i.e., beauty, pleasantness, and monotony vs. diversity), a one-way multivariate analysis of variance (MANOVA) was conducted. In case of significant omnibus tests indicating global differences across the qualitative sound rating dimensions, follow-up between group t-tests were conducted. Due to the exploratory nature of the study, no $p$-level correction was applied.

\section{Results}

\subsection{Experimental Results}

\subsubsection{Mood and paranoia}

The group*time interaction for depressive symptoms was highly significant, with a moderate effect size $\left(F(1,291)=11.28, p<.001, \eta^{2}\right.$ partial $\left.=.104\right)$. For state anxiety, the group*time interaction was highly significant and the effect was of moderate magnitude $(F(1,291)=10.40, p$ $<.001, \eta^{2}$ partial $\left.=.097\right)$. The group*time interaction for paranoia was highly significant and the effect was of moderate magnitude $\left(F(1,291)=7.35, p<.001, \eta^{2}\right.$ partial $\left.=.070\right)$. Repeating the analyses excluding cases that had one or both code numbers wrong did not change the significance or magnitude of the effects meaningfully.

\subsubsection{Cognition}

Concerning the two-error maximum length digit span forward, there was no significant time*group interaction effect $\left(F(1,281)=0.91, p=.437, \eta^{2}\right.$ partial $\left.=.010\right)$. The same null-finding emerged for the according backward digit span measure: group*time $(F(3,281)=1.09, p=.298$, $\left.\eta_{\text {partial }}^{2}=.004\right)$. For the n-back task total performance parameter, there were also consistent null- 
findings. There was no significant group*time interaction effect $\left(F(3,287)=0.59, p=.624, \eta^{2}\right.$ partial $=.006$ ). Accordingly, no significant interactions for any of the cognitive outcomes emerged after excluding the cases with a wrongly entered code, either. Similarly, an aggregation of both task scores into a composite $z$-score, which is an established practice in the field (Van Hedger et al., 2019), did not yield a significant result either. In the following post-hoc analyses are reported for the full sample.

\subsubsection{Post-hoc examination of significant interactions}

Post-hoc examination of the effects by computing within group dependent t-tests revealed that depressive symptoms significantly increased within both the low diversity urban soundscape $(T(1,82)=2.64, p=.010, d=0.29)$ and high diversity urban condition $(T(1,68)=4.88, p<.001$, $d=0.59$ ), whereas there were differential effects in the birdsong conditions with no change in the low diversity condition $(T(1,62)=-1.49, p=.142, d=-0.19)$ but a significant decrease in the high diversity condition $(T(1,60)=-2.57, p=.012, d=-0.29)$. For anxiety, there were no effects within both traffic noise conditions (low diversity: $T(1,82)=-1.37, p=.174, d=-0.15$; high diversity: $T(1,68)=0.49, p=.629, d=0.06)$, whereas there were significant declines in both birdsong conditions (low diversity: $T(1,62)=-6.13, p<.001, d=-0.77$; high diversity: $T(1,60)=-6.32, p$ $<.001, d=-0.70$ ). Finally, for paranoia there were no changes in the traffic noise conditions (low diversity: $T(1,82)=-0.55, p=.583, d=-0.06$; high diversity: $T(1,68)=0.67, p=.507, \mathrm{~d}=0.08)$, but significant decreases in both birdsong conditions (low diversity: $T(1,62)=-5.90, p<.001, d$ $=-0.74$; high diversity: $T(1,60)=-4.11, p<.001, d=-0.60)$. 

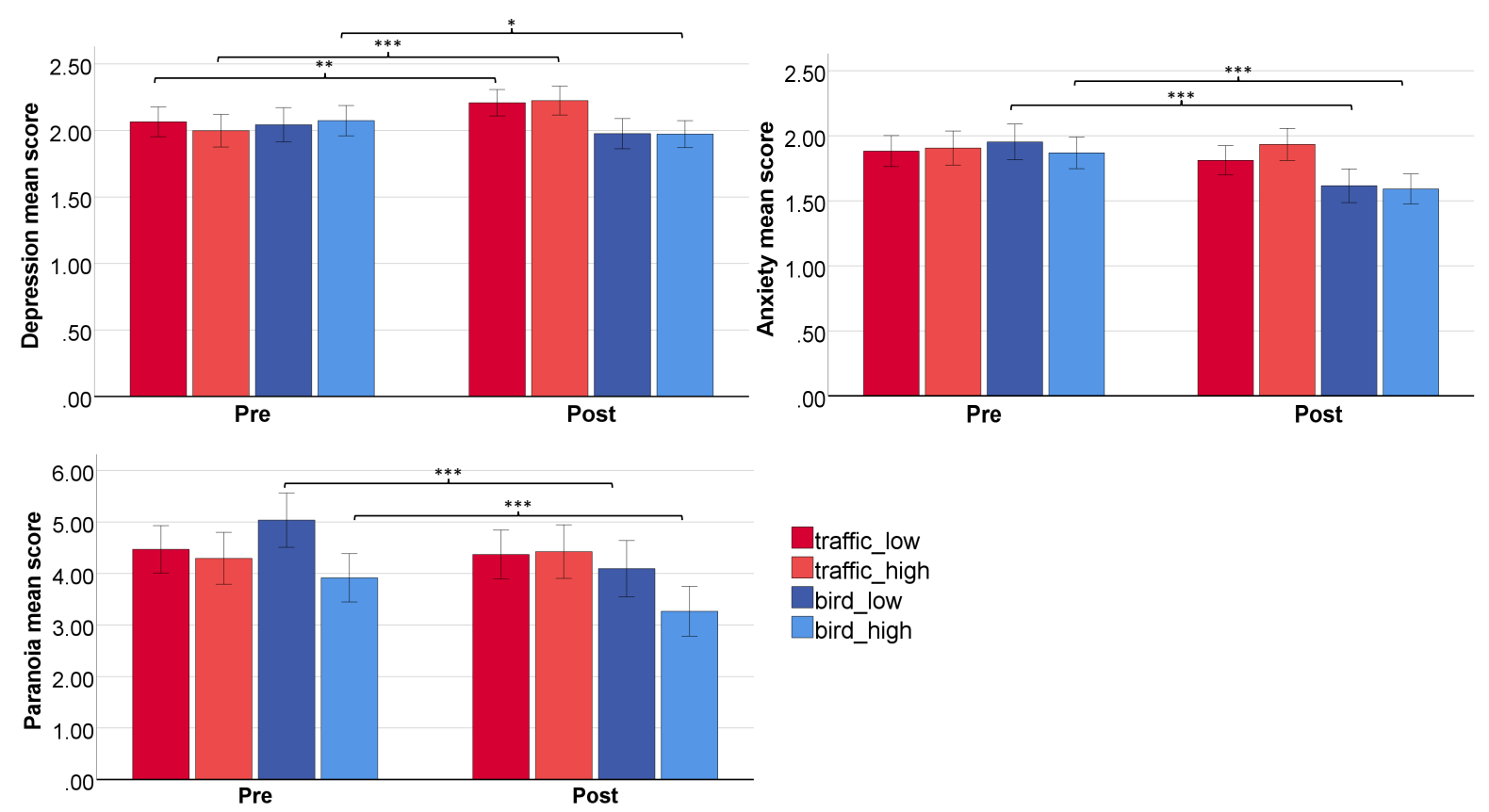

Figure 1.Effects of the different conditions on depressive symptoms (upper left), anxiety (upper right) and paranoia (lower left).

\subsubsection{Differences between low and high diversity conditions concerning mood and paranoia}

The low and high diverse traffic noise soundscapes were compared concerning magnitude of change $\left(\Delta_{\text {dep }}\right)$ in depressive symptoms. The t-test revealed no significant differences in change between these two conditions $(T(1,150)=1.13, p=.259$, Hedges' $g=0.19)$. For low vs. high diversity of the birdsong soundscapes concerning $\Delta_{\text {dep }}$, there was no significant difference either $(T(1,141)=0.58, p=.563$, Hedges' $g=0.10)$. Since for anxiety there were no effects in the traffic noise conditions, no further post-hoc tests were conducted. For the birdsong conditions, $\Delta_{\text {anx }}$ did not differ between the low- vs. high diversity groups $(T(1,141)=0.85, p=.297$, Hedges' $g=$ 0.14). Finally, also as there was no significant within-group change in the traffic noise conditions, only changes in the birdsong conditions $\left(\Delta_{\text {para }}\right)$ were compared for paranoia. Again, there was no 
PSYCHOLOGICAL EFFECTS OF BIRD VS. URBAN SOUNDSCAPES

difference in $\Delta_{\text {anx }}$ between the low and high diversity birdsong conditions $(T(1,141)=1.28, p=$ .202, Hedges' $g=0.22$ ).

\subsection{Perception of soundscapes}

The results of the MANOVA indicated a significant effect of condition on the perception (ratings) of soundscapes $(V=.495, F(9,834)=18.312, p<.001)$. Univariate follow-up ANOVAs revealed significant differences between the conditions regarding beauty $(F(3,278)=57.086, p<$ $.001)$ and pleasantness $(F(3,278)=62.394, p<.001)$ ratings of soundscapes but not concerning their monotony/ diversity $(F(3,278)=2.488, p=.061)$.

For statistical details, see Table 2. All traffic noise soundscapes (low and high diversity) differed significantly from all birdsong soundscapes (low and high diversity), i.e., beauty was always perceived as significantly higher for the birdsong conditions in any given comparison with the traffic noise conditions (all $p<.001$ ), with large effect sizes. No significant effects were found for the perception of beauty within the categories of traffic noise or birdsongs (low diversity vs. high diversity). For the perceived pleasantness, high diversity traffic noise was the least pleasant, significantly differing from low diversity traffic noise $(p<.05)$. The birdsong conditions again did not significantly differ from one another. The traffic noise soundscapes in general were perceived as significantly less pleasant compared to the birdsong soundscapes in any given comparison (all $p<.001$ ). Concerning perceived monotony/ diversity, a significant difference was revealed between the low diversity traffic noise and high diversity traffic noise soundscapes $(p<.05)$ indicating that highly diverse traffic noise is perceived as more diverse/ less monotone compared to low diversity traffic noise. 
PSYCHOLOGICAL EFFECTS OF BIRD VS. URBAN SOUNDSCAPES
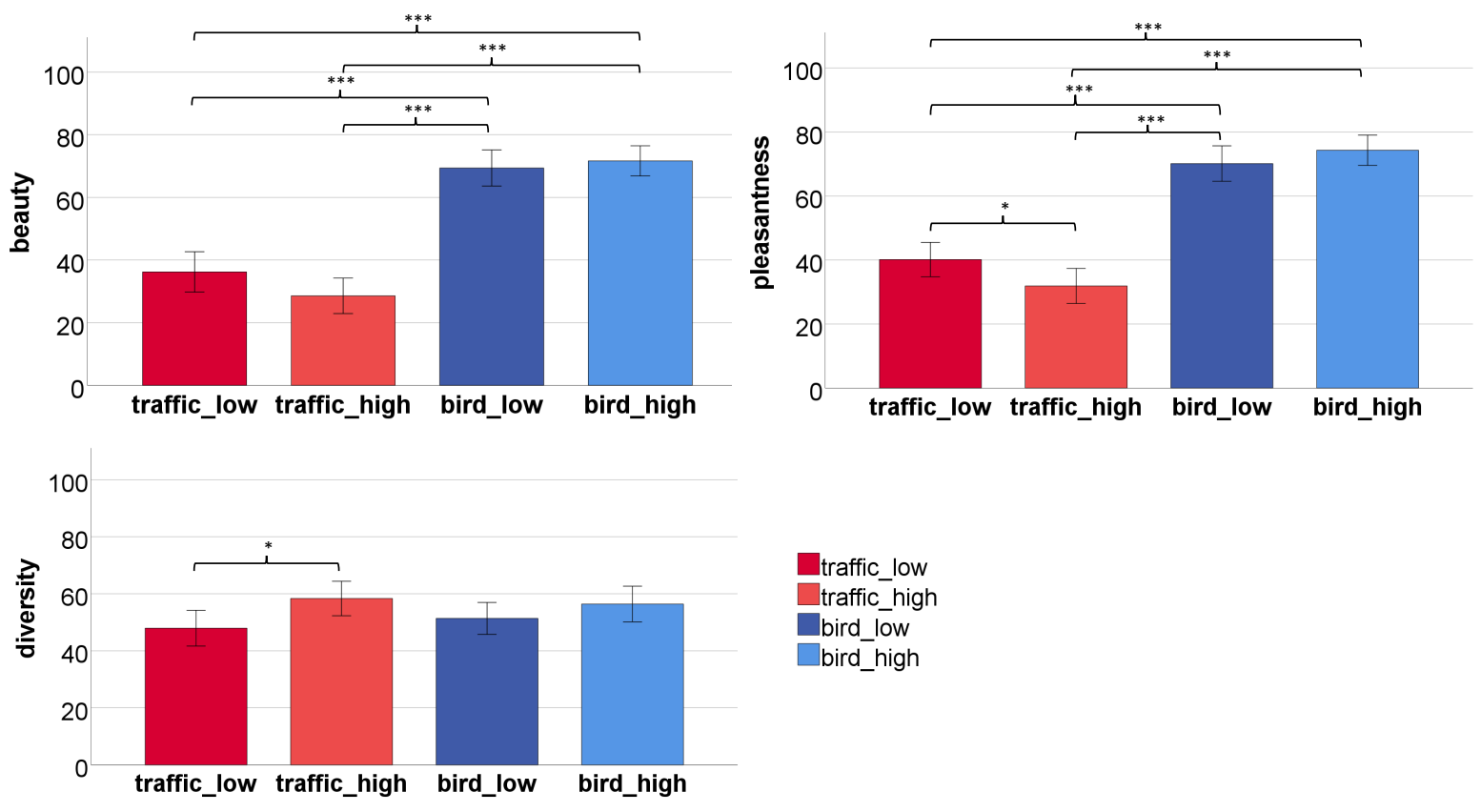

Figure 2.Participants' average ratings of the soundscape per group. Ratings for beauty (upper left), pleasantness (upper right) and diversity (lower left) are shown. 
Table 2.Comparison of soundscape ratings concerning beauty, pleasantness and diversity

\begin{tabular}{|c|c|c|c|c|}
\hline DV & Conditions & $t(\mathrm{df})$ & $p$-value & Cohen's $d$ \\
\hline \multicolumn{5}{|c|}{ Beauty } \\
\hline & $\begin{array}{l}\text { Traffic noise low vs. } \\
\text { traffic noise high }\end{array}$ & $1.768(146.956)$ & .079 & .284 \\
\hline & $\begin{array}{l}\text { Traffic noise low vs. } \\
\text { birdsong low }\end{array}$ & $-7.648(141.826)$ & $.000 * * *$ & -1.238 \\
\hline & $\begin{array}{l}\text { Traffic noise low vs. } \\
\text { birdsong high }\end{array}$ & $-8.754(146.230)$ & $.000 * * *$ & -1.375 \\
\hline & $\begin{array}{l}\text { Traffic noise high vs. } \\
\text { birdsong low }\end{array}$ & $-10.081(125)$ & $.000 * * *$ & -1.791 \\
\hline & $\begin{array}{l}\text { Traffic noise high vs. } \\
\text { birdsong high }\end{array}$ & $-11.603(136)$ & $.000 * * *$ & -1.977 \\
\hline & $\begin{array}{l}\text { Birdsong high vs. } \\
\text { birdsong low }\end{array}$ & $.606(131)$ & .546 & .105 \\
\hline \multicolumn{5}{|c|}{ Pleasantness } \\
\hline & $\begin{array}{l}\text { Traffic noise low vs. } \\
\text { traffic noise high }\end{array}$ & $2.138(147)$ & $.036^{*}$ & .349 \\
\hline & $\begin{array}{l}\text { Traffic noise low vs. } \\
\text { birdsong low }\end{array}$ & $-7.596(142)$ & $.000 * * *$ & -1.281 \\
\hline & $\begin{array}{l}\text { Traffic noise low vs. } \\
\text { birdsong high }\end{array}$ & $-9.501(152.483)$ & $.000 * * *$ & -1.509 \\
\hline & $\begin{array}{l}\text { Traffic noise high vs. } \\
\text { birdsong low }\end{array}$ & $-9.775(125)$ & $.000 * * *$ & -1.736 \\
\hline & $\begin{array}{l}\text { Traffic noise high vs. } \\
\text { birdsong high }\end{array}$ & $-11.724(136)$ & $.000 * * *$ & -1.998 \\
\hline & $\begin{array}{l}\text { Birdsong high vs. } \\
\text { birdsong low }\end{array}$ & $1.151(131)$ & .252 & .200 \\
\hline \multicolumn{5}{|c|}{$\begin{array}{l}\text { Monotony/ } \\
\text { diversity }\end{array}$} \\
\hline & $\begin{array}{l}\text { Traffic noise low vs. } \\
\text { traffic noise high }\end{array}$ & $-2.381(146.295)$ & $.019 *$ & -.386 \\
\hline & $\begin{array}{l}\text { Traffic noise low vs. } \\
\text { birdsong low }\end{array}$ & $-.812(141.828)$ & .418 & -.131 \\
\hline & $\begin{array}{l}\text { Traffic noise low vs. } \\
\text { birdsong high }\end{array}$ & $-1.893(153)$ & .060 & -.305 \\
\hline & $\begin{array}{l}\text { Traffic noise high vs. } \\
\text { birdsong low }\end{array}$ & $1.687(125)$ & .094 & .300 \\
\hline & $\begin{array}{l}\text { Traffic noise high vs. } \\
\text { birdsong high }\end{array}$ & $.446(136)$ & .657 & .076 \\
\hline & $\begin{array}{l}\text { Birdsong high vs. } \\
\text { birdsong low }\end{array}$ & $1.181(131)$ & .240 & .206 \\
\hline
\end{tabular}




\section{Discussion}

The present study demonstrated that non-diverse (small effect size) and highly diverse (medium effect size) traffic noise soundscapes aggravated depressive states, whereas the highly diverse birdsong soundscape decreased depressive states (small effect size). Concerning anxiety, traffic noise soundscapes had no effect, whereas both birdsong soundscapes significantly alleviated anxiety (medium effect size). Finally, the traffic noise soundscapes had no effect on paranoia, whereas again both birdsong soundscapes significantly lowered it (medium effect size). The beneficial effects of birdsongs in particular concerning mood and attention restoration have been previously observed (Ratcliffe et al., 2013). Mood recovery (e.g., after a stressor) or beneficial mood effects have repeatedly been reported for exposure with natural sounds (Benfield et al., 2014; Jiang et al., 2021). The present study thus confirms prior findings. Moreover, to the best of our knowledge, beneficial effects of natural soundscapes on state paranoia are shown for the very first time. This finding might be explained in several ways. Birdsongs might be implicitly associated with a vital natural environment, divert attention away from (internal and external) stressors, or could signal the absence of acute threat. Urban soundscapes on the other hand might trigger socio-evaluative concerns, involuntarily direct attention resulting in perceived loss of control and hence alter vigilance to potential threats which are processes proposed to elicit paranoia (20). However, somewhat contradicting the latter notion, the traffic noise soundscapes as used in the present study did not increase paranoia. Possibly, by adding human voices to the audio file, this effect could have been evoked. Human voices may more readily activate interpersonal sensitivity, to which a central role has been ascribed in the emergence and maintenance of paranoia (Meisel et al., 2018). 


\section{PSYCHOLOGICAL EFFECTS OF BIRD VS. URBAN SOUNDSCAPES}

Neither urban (traffic noise) nor natural (birdsong) soundscapes had any effect on cognitive performance, which, at first glance, seems to contradict a previous study, which implemented the same cognitive tests (i.e., digit span and dual n-back) (Van Hedger et al., 2019). An explanation for the null effect could be the degree to which the administration of the tasks was controlled. The current study was performed online, practice was restricted to two blocks (i.e., in a laboratory setting, participants can often train for as long as they wish/need), and hence there was little control over the degree to which subjects understood the task correctly, albeit visual inspection of the raw data did not reveal severe deviations from an expected performance. In addition, the online as opposed to a laboratory situation does not allow for controlling context variables or systematically manipulating baseline levels of stress or fatigue (Benfield et al., 2014).

\subsection{Limitations}

One limitation of the present study is the numerically higher percentage of males relative to females (albeit non-significant), which is a typical problem encountered in online studies. Future studies should stratify the subgroups by sex, such as to balance the sample in this regard. Furthermore, the manipulation of low vs. high diversity of the soundscapes was only partially successful. Namely, although the soundscape composition followed a logical rationale in this regard, and the subjective diversity rating the urban soundscapes significantly differed in the traffic noise soundscapes, this was not the case for the birdsong conditions. To assure stronger contrasts, and hence to be able to test the diversity hypothesis more aptly concerning enhanced beneficial effects, the contrast between the soundscapes needs to be further enhanced in future studies. In addition, mixed conditions such as urban soundscapes containing birdsongs would be a highly interesting research target, as this could more readily reflect daily life exposure situations. Finally, 
although the instructions required participants to set their audio system loudness to $80 \%$, still subjectively perceived loudness of the soundscapes could constitute a confounding factor.

\subsection{Conclusion}

The present study provides evidence for the beneficial effects of birdsongs on mood (depression, anxiety) and paranoid symptoms, with the latter being shown for the first time. On the other hand, the negative effects of traffic noise were only confirmed concerning depressive symptoms. The manipulated low vs. high diversity of the soundscapes did not have a significant effect, which might in part be explained by no perceived subjective differences concerning monotony vs. diversity. Further replication in vulnerable, elevated risk- or clinical groups could be of interest to assess the magnitude of effects given pre-existing symptoms and cognitive performance deficits. In case of replication, using birdsongs as 'soothing' background soundscape could open interesting new possibilities in psychiatric hospital or other therapeutic settings. 


\section{Acknowledgements}

We would like to thank Armin Junge for assisting us in creating the stimuli for this study. Furthermore, we thank Nicolás Ascone Michelis for his help with optimizing the birdsong soundscapes

\section{Ethic statement}

All procedures performed in studies involving human participants were in accordance with the ethical standards of the institutional and/or national research committee and with the 1964 Helsinki Declaration and its later amendments or comparable ethical standards. Informed consent was obtained from all participating subjects.

\subsection{Declaration of conflicting interest}

None.

\subsection{Funding and role of funders}

This study was not externally funded. 


\section{References}

UN (2018). $68 \%$ of the world population projected to live in urban areas by 2050 , says UN | UN DESA | United Nations Department of Economic and Social Affairs. (2018, May 16). https://www.un.org/development/desa/en/news/population/2018-revision-of-worldurbanization-prospects.html

Benfield, J. A., Taff, B. D., Newman, P., \& Smyth, J. (2014). Natural Sound Facilitates Mood Recovery. Ecopsychology, 6(3), 183-188. https://doi.org/10.1089/eco.2014.0028

Berman, M. G., Jonides, J., \& Kaplan, S. (2008). The cognitive benefits of interacting with nature. Psychological Science, 19(12), 1207-1212. https://doi.org/10.1111/j.14679280.2008.02225.x

Freeman, D., Garety, P. A., Bebbington, P. E., Smith, B., Rollinson, R., Fowler, D., Kuipers, E., Ray, K., \& Dunn, G. (2005). Psychological investigation of the structure of paranoia in a non-clinical population. The British Journal of Psychiatry, 186(5), 427-435. https://doi.org/10.1192/bjp.186.5.427

Hedblom, M., Knez, I., \& Gunnarsson, B. (2017). Bird Diversity Improves the Well-Being of City Residents. Ecology and Conservation of Birds in Urban Environments, 287-306. https://doi.org/10.1007/978-3-319-43314-1_15

Inquisit 5 [Computer software]. (2016). Retrieved from https://www.millisecond.com.

Jaeggi, S. M., Studer-Luethi, B., Buschkuehl, M., Su, Y.-F., Jonides, J., \& Perrig, W. J. (2010). The relationship between n-back performance and matrix reasoning-Implications for training and transfer. Intelligence, 38(6), 625-635.

Jiang, B., Xu, W., Ji, W., Kim, G., Pryor, M., \& Sullivan, W. C. (2021). Impacts of nature and built acoustic-visual environments on human's multidimensional mood states: A cross- 
continent experiment. Journal of Environmental Psychology, 77, 101659.

https://doi.org/10.1016/j.jenvp.2021.101659

Krabbendam, L. (2005). Schizophrenia and Urbanicity: A Major Environmental Influence-Conditional on Genetic Risk. Schizophrenia Bulletin, 31(4), 795-799. https://doi.org/10.1093/schbul/sbi060

Laux, L., Hock, M., Bergner-Köther, R., Hodapp, V., Renner, K.-H., Laux, L., Hock, M., Bergner-Köther, R., Hodapp, V., \& Renner, K. H. (2016). STADI. Diagnostische Verfahren in Der Psychotherapie, 1, 478.

Lincoln, T. M., Peter, N., Schäfer, M., \& Moritz, S. (2010). From stress to paranoia: An experimental investigation of the moderating and mediating role of reasoning biases. Psychological Medicine, 40(1), 169-171. https://doi.org/10.1017/S003329170999095X

Meisel, S. F., Garety, P. A., Stahl, D., \& Valmaggia, L. R. (2018). Interpersonal processes in paranoia: A systematic review. Psychological Medicine, 48(14), 2299-2312. https://doi.org/10.1017/S0033291718000491

Mossaheb, N., Becker, J., Schaefer, M. R., Klier, C. M., Schloegelhofer, M., Papageorgiou, K., \& Amminger, G. P. (2012). The Community Assessment of Psychic Experience (CAPE) questionnaire as a screening-instrument in the detection of individuals at ultra-high risk for psychosis. Schizophrenia Research, 141(2-3), 210-214. https://doi.org/10.1016/j.schres.2012.08.008

Penkalla, A. M., \& Kohler, S. (2014). Urbanicity and Mental Health in Europe: A Systematic Review. European Journal of Mental Health, 9(2), 163-177. https://doi.org/10.5708/EJMH.9.2014.2.2 
Ratcliffe, E., Gatersleben, B., \& Sowden, P. T. (2013). Bird sounds and their contributions to perceived attention restoration and stress recovery. Journal of Environmental Psychology, 36, 221-228. https://doi.org/10.1016/j.jenvp.2013.08.004

Ritchie, H., \& Roser, M. (2018). Urbanization. Our World in Data. https://ourworldindata.org/urbanization

Sampson, L., Ettman, C. K., \& Galea, S. (2020). Urbanization, urbanicity, and depression: A review of the recent global literature. Current Opinion in Psychiatry, 33(3), 233-244. https://doi.org/10.1097/YCO.0000000000000588

Schlier, B., Jaya, E. S., Moritz, S., \& Lincoln, T. M. (2015). The Community Assessment of Psychic Experiences measures nine clusters of psychosis-like experiences: A validation of the German version of the CAPE. Schizophrenia Research, 169(1), 274-279. https://doi.org/10.1016/j.schres.2015.10.034

Schlier, B., Moritz, S., \& Lincoln, T. M. (2016). Measuring fluctuations in paranoia: Validity and psychometric properties of brief state versions of the Paranoia Checklist. Psychiatry Research, 241, 323-332. https://doi.org/10.1016/j.psychres.2016.05.002

Seidler, M., Romero-Starke, A., Zeeb, H., Riedel-Heller, S., \& Hegewald. (2019). Traffic noise and mental illness - a systematic review: Environmental Epidemiology, 3, 360. https://doi.org/10.1097/01.EE9.0000609984.76129.9e

Ulrich, R. S. (1983). Aesthetic and Affective Response to Natural Environment. In I. Altman \& J. F. Wohlwill (Eds.), Behavior and the Natural Environment (pp. 85-125). Springer US. https://doi.org/10.1007/978-1-4613-3539-9_4

Van Hedger, S. C., Nusbaum, H. C., Clohisy, L., Jaeggi, S. M., Buschkuehl, M., \& Berman, M. G. (2019). Of cricket chirps and car horns: The effect of nature sounds on cognitive 
performance. Psychonomic Bulletin \& Review, 26(2), 522-530.

https://doi.org/10.3758/s13423-018-1539-1

Woods, D. L., Kishiyama, M. M., Yund, E. W., Herron, T. J., Edwards, B., Poliva, O., Hink, R. F., \& Reed, B. (2011). Improving digit span assessment of short-term verbal memory. Journal of Clinical and Experimental Neuropsychology, 33(1), 101-111. https://doi.org/10.1080/13803395.2010.493149 


\section{Appendix}

Supplementary Table 1. Descriptive pre-post data of main outcomes for all conditions

\begin{tabular}{|c|c|c|c|c|c|c|c|c|c|c|}
\hline \multirow[t]{2}{*}{ Category } & \multirow[t]{2}{*}{ Variable } & & \multicolumn{2}{|c|}{$\begin{array}{c}\text { Traffic } \\
\text { noise low } \\
\mathbf{n}=\mathbf{8 3}\end{array}$} & \multicolumn{2}{|c|}{$\begin{array}{c}\text { Traffic } \\
\text { noise high } \\
\text { n }=69\end{array}$} & \multicolumn{2}{|c|}{$\begin{array}{c}\text { Birdsong } \\
\text { low } \\
n=63\end{array}$} & \multicolumn{2}{|c|}{$\begin{array}{c}\text { Birdsong } \\
\text { high } \\
\mathbf{n}=\mathbf{8 0}\end{array}$} \\
\hline & & & $M$ & $S D$ & $M$ & $S D$ & $M$ & $S D$ & $M$ & $S D$ \\
\hline \multicolumn{11}{|l|}{ Mood } \\
\hline \multirow[t]{7}{*}{ Depression } & & Pre & 2.06 & 0.57 & 1.99 & 0.53 & 2.04 & 0.42 & 2.07 & 0.50 \\
\hline & & Post & 2.20 & 0.44 & 2.22 & 0.53 & 1.97 & 0.40 & 1.97 & 0.43 \\
\hline & A & Pre & 1.88 & 0.54 & 1.90 & 0.62 & 1.95 & 0.55 & 1.86 & 0.48 \\
\hline & & Post & 1.81 & 0.53 & 1.93 & 0.63 & 1.61 & 0.43 & 1.59 & 0.47 \\
\hline & Paranoia & & & & & & & & & \\
\hline & & Pre & 4.46 & 2.41 & 4.29 & 2.25 & 5.03 & 1.94 & 3.91 & 1.82 \\
\hline & & Post & 4.36 & 2.23 & 4.42 & 2.34 & 4.09 & 2.08 & 3.26 & 2.01 \\
\hline
\end{tabular}

\section{Cognition}

Digit span

(max 2 errors)

forward

$\begin{array}{lllllllll}\text { Pre } & 7.43 & 2.38 & 7.17 & 2.04 & 7.16 & 2.25 & 7.56 & 2.58 \\ \text { Post } & 7.33 & 2.25 & 7.70 & 2.41 & 7.23 & 3.23 & 7.67 & 2.69\end{array}$

Digit span max length forward

$\begin{array}{lllllllll}\text { Pre } & 8.13 & 2.19 & 7.98 & 1.79 & 8.18 & 2.27 & 8.23 & 2.32 \\ \text { Post } & 7.98 & 1.93 & 8.21 & 2.30 & 8.18 & 2.96 & 8.39 & 2.50\end{array}$

Digit span (max 2 errors) backwards

$\begin{array}{lllllllll}\text { Pre } & 6.66 & 2.50 & 6.74 & 2.09 & 6.52 & 2.76 & 6.51 & 2.30 \\ \text { Post } & 6.61 & 2.12 & 6.67 & 2.32 & 7.03 & 3.14 & 6.41 & 2.65\end{array}$

Digit span max $\begin{array}{lllllllll}\text { Post } & 6.61 & 2.12 & 6.67 & 2.32 & 7.03 & 3.14 & 6.41 & 2.65\end{array}$

length backward

$\begin{array}{lllllllll}\text { Pre } & 7.35 & 2.13 & 7.53 & 1.81 & 7.28 & 2.63 & 7.24 & 2.14\end{array}$

$\begin{array}{lllllllll}\text { Post } & 7.27 & 2.04 & 7.59 & 2.11 & 7.59 & 2.85 & 7.39 & 2.54\end{array}$

n-back

$\begin{array}{lllllllll}\text { Pre } & .705 & 1.73 & 1.15 & 1.87 & .502 & 2.14 & .667 & 2.60 \\ \text { Post } & .822 & 1.78 & 1.20 & 1.98 & .371 & 2.52 & .819 & 2.75\end{array}$


PSYCHOLOGICAL EFFECTS OF BIRD VS. URBAN SOUNDSCAPES

Supplementary Table 2. Paired t-test statistics for changes within groups in mood and paranoia

\begin{tabular}{lllll}
\hline Condition & Variable & \multicolumn{2}{l}{ Inferential statistics } \\
\hline & & $\mathrm{T}(\mathrm{df})$ & $\mathrm{p}$-value & Cohen's d \\
\cline { 3 - 4 } Traffic noise & Depression & $2.64(82)$ & $.010^{*}$ & 0.29 \\
low & Anxiety & $-1.37(82)$ & .174 & -0.15 \\
$\mathbf{n = 8 3}$ & Paranoia & $-0.55(82)$ & .583 & -0.06 \\
& & & & \\
Traffic noise & Depression & $4.88(68)$ & $.000^{* * *}$ & 0.59 \\
high & Anxiety & $0.49(68)$ & .629 & 0.06 \\
$\mathbf{n}=\mathbf{6 9}$ & Paranoia & $0.67(68)$ & .507 & .080 \\
& & & & \\
Birdsong low & Depression & $-1.49(62)$ & .142 & -0.19 \\
$\mathbf{n}=\mathbf{6 3}$ & Anxiety & $-6.13(62)$ & $.000^{* * *}$ & -0.77 \\
& Paranoia & $-5.90(62)$ & $.000^{* * *}$ & -0.74 \\
& & & & \\
Birdsong high & Depression & $-2.57(60)$ & $.012^{*}$ & -0.29 \\
$\mathbf{n = 8 0}$ & Anxiety & $-6.32(60)$ & $.000^{* * *}$ & -0.70 \\
& Paranoia & $-4.11(60)$ & $.000^{* * *}$ & -0.60 \\
\hline
\end{tabular}




\section{Supplementary Figure 1.}

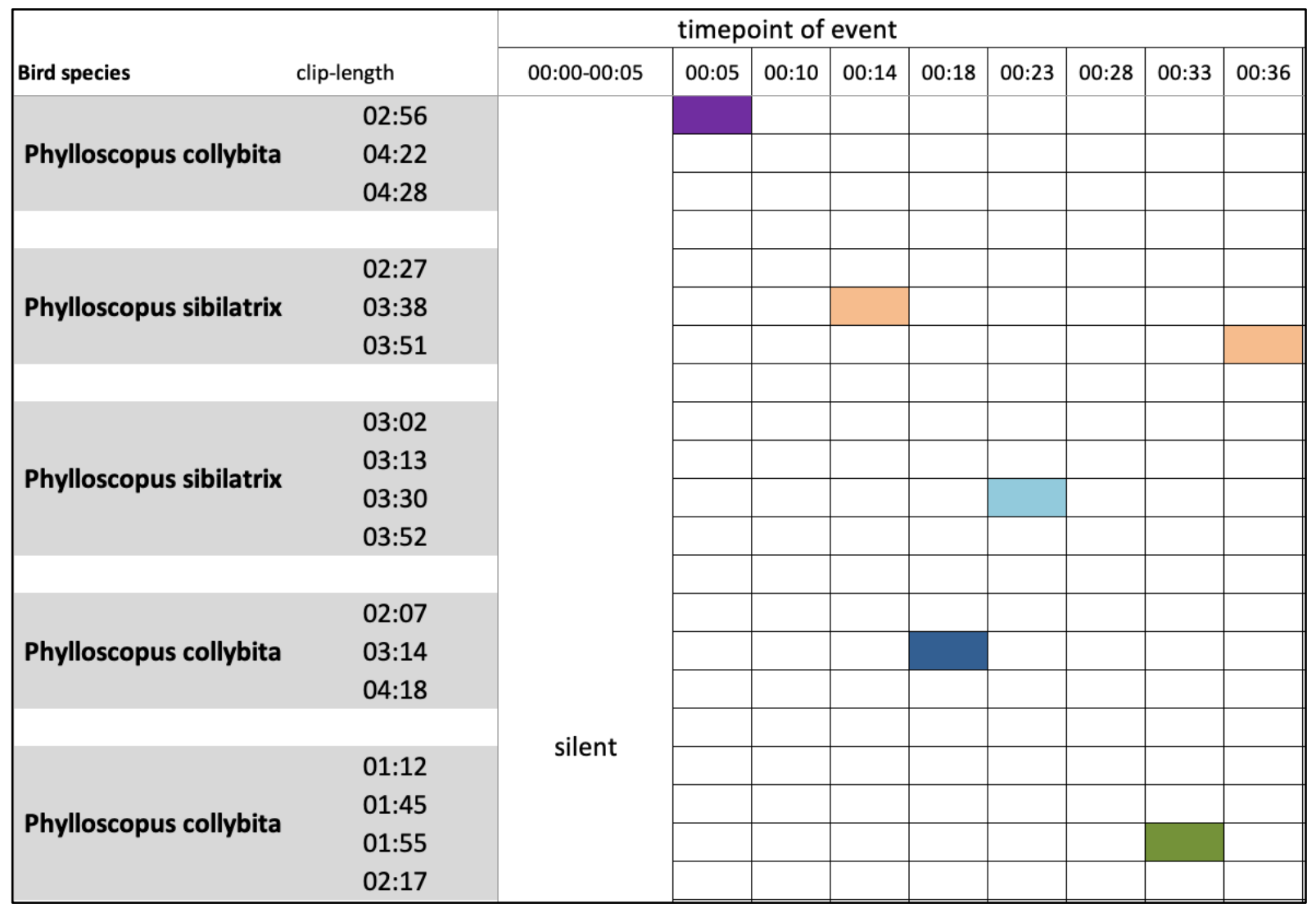

Supplementary Figure 1. Depicted here is an example on how the low diversity birdsong condition was generated. On the left the participating bird species are listed and on the right a flow of events is shown 